\title{
Tipos de materiales
}

\author{
Types of materials
}

\author{
Martha L. Ignacio-Martínez ${ }^{a}$, Sbeydi L. Vázquez-Flores ${ }^{b}$, Denisse Cruz-Castellanos ${ }^{c}$
}

\begin{abstract}
:
The constant contact with materials through objects and tools, has created curiosity about their importance of study, so this research presents the bases to understand the behavior of materials through the relationship between their structure, properties and characteristics, facilitating the recognition of these in practical applications in the field of work. The concept of material is approached, which refers to a set of elements present in space, where its behavior is defined by its structure at the microscopic level, which determines its atomic link by attributing the properties to the material. Among these properties are physical, chemical and mechanical; that describe the behavior of the material to various stimulus.
\end{abstract}

Keywords:

Materials, properties, bands, elements

\section{Resumen:}

El constante contacto con los materiales a través de objetos y herramientas, ha generado curiosidad por su importancia de estudio, por lo que en esta investigación se presentan las bases para comprender el comportamiento de los materiales a través de la relación existente entre su estructura, propiedades y características, facilitando el reconocimiento de estos en aplicaciones prácticas en el campo laboral. Se aborda el concepto de material, el cual hace referencia a un conjunto de elementos presentes en el espacio, donde su comportamiento está definido por su estructura a nivel microscópica, la cual determina su enlace atómico atribuyendo las propiedades al material. Dentro de estas propiedades se encuentran las físicas, químicas y mecánicas; que describen el comportamiento del material ante diversos estímulos.

\section{Palabras Clave:}

Material, propiedades, bandas, elementos

\section{Introducción}

Un material es un conjunto de elementos que, posee forma, peso, volumen y un lugar en el espacio; es la materia física que puede ser manipulada y empleada por el hombre, generalmente sin que se produzca una conversión química mayoritaria durante su función.

Las propiedades dependen principalmente de su estructura atómica, su estructura cristalina y su microestructura. Estas propiedades permiten determinar su comportamiento ante estímulos externos.

A continuación, se mencionan algunas de las propiedades que describen a los materiales:

\section{- Propiedades físicas:}

Describen el comportamiento del material que se basa en la estructura, sustancia o materia que es visible y medible. Por ejemplo, el peso, color, resistencia eléctrica, etc.

- Propiedades químicas:

Describen cómo se comporta el material al estar en contacto con sustancias químicas.

- Propiedades mecánicas:

Describen cómo se comporta el material al aplicarfuerzas externas.

\footnotetext{
Autor de correspondencia, Universidad Autónoma del Estado de Hidalgo, https://orcid.org/0000-0002-3424-7525, Email: ig337033@uaeh.edu.mx

b Universidad Autónoma del Estado de Hidalgo, https://orcid.org/0000-0002-4801-9523, Email: va353898@uaeh.edu.mx

c Universidad Autónoma del Estado de Hidalgo, https://orcid.org/0000-0002-1126-8091, Email: cr420686@uaeh.edu.mx
} 


\section{Tipos de materiales}

El estudio de materiales conlleva a una clasificación, la cual comienza con la teoría de ban das de Heitlery London de acuerdo con su conductividad; permitiendo una explicación cualitativa más clara de los materiales. [1]

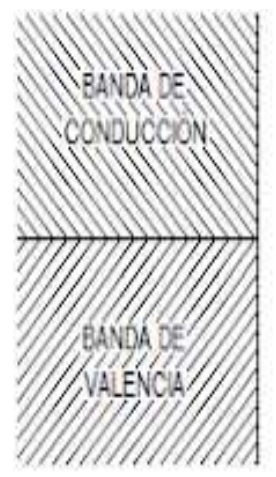

CONDUCTOR

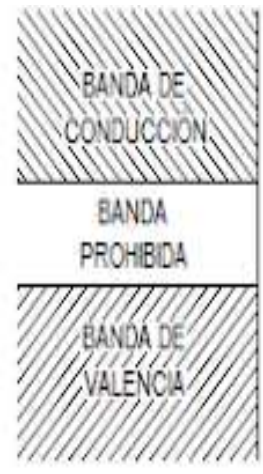

SEMICONDUCTOR

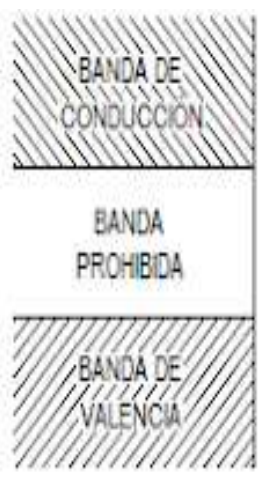

ALLANTE
Figura 1. Los materiales, de acuerdo a su conductividad, pueden clasificarse en tres categorías principales: conductores, semiconductores y aislantes. [1]

\section{Metales}

Su estructura electrónica les permite conducir la corriente eléctrica a bajas temperaturas o temperatura ambiente. La resistividad al paso de la corriente eléctrica es baja, y la banda de valencia se encuentra próxima a la de conducción. Esta resistividad crece con la temperatura. $[1,2]$

Sus átomos se encuentran unidos mediante enlaces metálicos. Forman aleaciones mediante un proceso de fundición y solidificación: ferrosas y no ferrosas. Son de alta densidad y suelen ser maleables. Tienen una resistencia mecánica y su estructura es cristalina. Todos son sólidos en condiciones naturales excepto el mercurio (brillo metálico). [3,4]

Algunos elementos metálicos son el oro, la plata y el magnesio. Estos materiales pueden ser aplicables en la producción de espejos astronómicos, de acero y construcciones, por ejemplo, en un puente. [5]

\section{Semiconductor}

Cuerpo en el que el intervalo de energía entre la banda normal y la banda de excitación próxima es del mismo orden de magnitud que en los semiconductores intrínsecos, presenta niveles intermedios susceptibles situados a la banda prohibida, debido a la presencia de impurezas. La banda prohibida es de aproximadamente 1 eV y se crea un hueco que actúa como un transportador de carga positiva cuando se transfiere el electrón a la banda de conducción.
El germanio, silicio y azufre son algunos ejemplos de materiales semiconductores. [1,2]

Existen dos tipos:

- Intrínsecos:Semiconductor puro.

- Extrínsecos:al dopar o añadir átomos de impureza a un semiconductor puro para aumentar su conductividad se convierte en extrínseco. Tienen 2 tipos de impurezas (átomo) de tipo: [1,2,6,7]

- $\quad \mathrm{p}$ : Impurezas aceptores trivalentes.

Material aceptor.

Crea abundancia de huecos.

Menos electrones (portadores minoritarios).

- $\mathrm{n}$ : son impurezas donadoras pentavalentes.

Más electrones (portadores mayoritarios).

\section{Cerámicos}

Cuerpo en el que la banda normal está llena y separada de la primera banda de excitación por una banda prohibida bastante ancha. Esta banda prohibida es el orden de varios electrón-voltios. Existe una barrera de energía de $6 \mathrm{eV}$, la conductibilidad es pequeña porque solo un número reducido de electrones sobrepasa el intervalo prohibido. Para que los electrones puedan pasar a la banda de conducción se necesita una gran energía, la cual rompería al material. [1,2]

Pueden ser cristalinos o amorfos, tienen un elevado punto de fusión, una elevada dureza y resistencia. Presentan baja densidad, baja ductilidad, confortabilidad y resistencia al impacto. [4,5]

Son utilizados en la producción de vidrio, productos de arcillas, refractarios, abrasivos, cementos, etcétera.

\section{Polímeros}

Son compuestos orgánicos basados en elementos como el carbono, hidrógeno y otros elementos no metálicos. Están formados por cadenas largas de monómeros unidos entre sí, que presentan baja densidad, elevado peso molecular, son resistentes a la corrosión, son flexibles, tienen alta resistencia mecánica y son aislantes eléctricos. [4,5]

Los plásticos, el caucho, el almidón, la celulosa y la seda son algunos ejemplos de estos materiales; los cuales son empleados para la fabricación de juguetes, artículos domésticos, neumáticos, etc.

Existen 3 tipos de plásticos: termoplásticos (son relativamente blandos y dúctiles, por ejemplo: piezas pequeñas en aparatos), termoestable (son compactos y duros, tienen temperaturas de fusión relativamente altas, un ejemplo de ellos es la melanina) y elastómeros (permiten obtener plásticos de gran elasticidad que 
recuperan su forma y sus dimensiones cuando deja de actuar sobre ellos una fuerza). [4,8]

\section{Biomateriales}

Son una sustancia creada por un organismo vivo o que está en contacto con él. Presentan propiedades mecánicas como: ductilidad, elasticidad, dureza y resistencia a la atracción. Estos materiales deben ser biocompatibles, biodegradables (dependiendo al organismo) y no deben ser tóxicos ni cancerígenos. [9, 10,11]

Su aplicación tiene cabida en los implantes médicos, tejidos humanos regenerados, biosensores y en las prótesis.

Hay dos tipos de biomateriales de acuerdo a su origen: [11]

- Naturales: colágeno, lana y seda.

- Sintéticos: comúnmente denominados materiales biomédicos, ejemplo: polímeros, cerámicos, metálicos y materiales compuestos.

\section{Materiales inteligentes}

Es un grupo de materiales donde una o varias de sus propiedades pueden ser modificadas bajo estímulos externos (luz, calor, humedad, cambios de Ph, fuerza mecánica, etc.) Se identifican como materiales que por su microestructura pueden comportarse como sensores actuadores y controladores. Estos materiales pueden utilizarse para generación de nuevas tecnologías, para generar energía limpia, creación de sensores de temperatura y corrosión, así como en aparatos de ortodoncia. [12,13]

Algunos materiales inteligentes son: [12,14]

- Piezoeléctricos: pueden convertirse en energía mecánica a eléctrica y viceversa.

- Memoria de forma: poseen la capacidad de cambiar de forma, incluso devolver a su forma original.

- Cromoactivos: cambian de color al ser sometidos a una variación de temperatura, luz, presión, etc.

- Magnetorreológicos: cambian sus propiedades ante un campo magnético.

- Focoactivos. 


\section{MATERJAL}

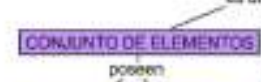

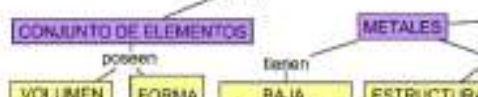

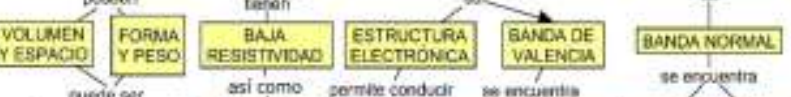

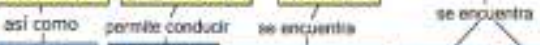

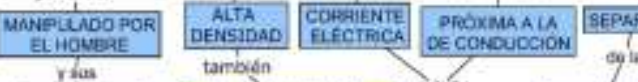
Tras

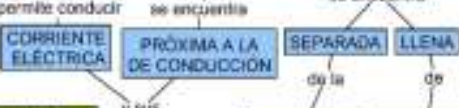
son depanden

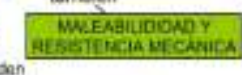

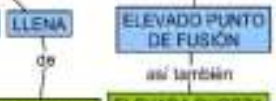

(n) oil

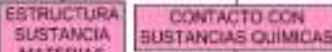

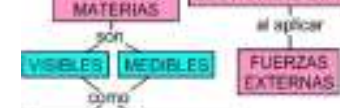

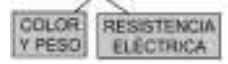

\section{astic
unison por \\ periun}

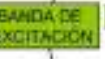

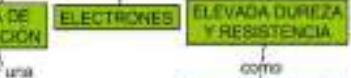

Merincicos

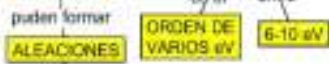
de tipe

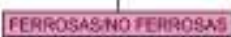

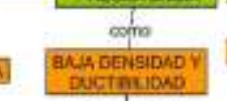
por ke que san EMPLEADOS PARA
PRODUCCSON DE VIDRO

$$
\begin{gathered}
1 \\
x \text { en } \\
\hline \text { propucos }
\end{gathered}
$$

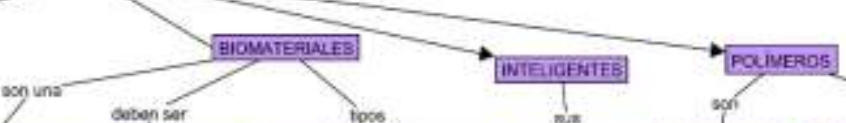
Sistencos Mos

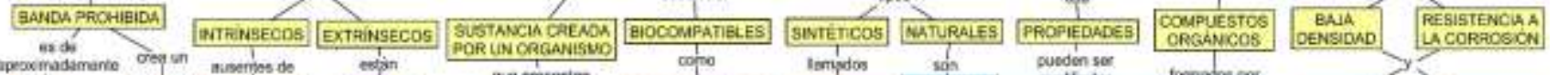

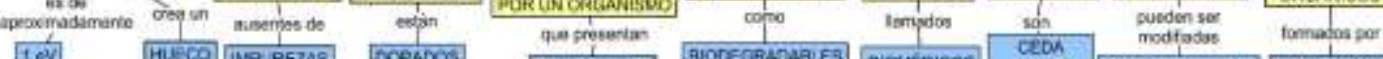

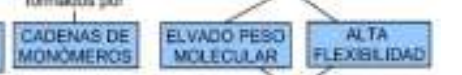

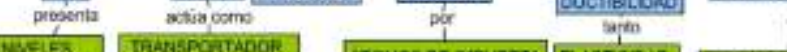

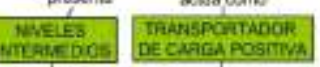

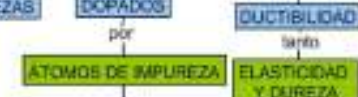
wer ron

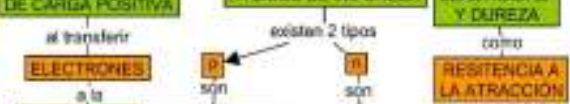
MPIPEz-245

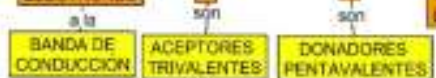

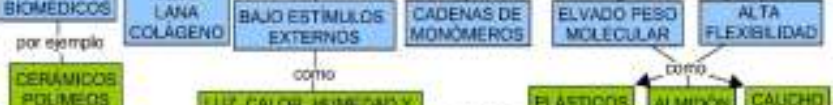

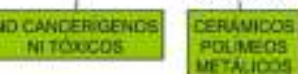

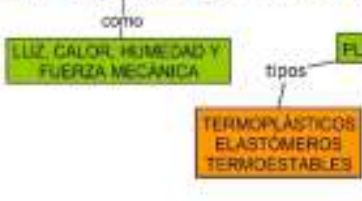

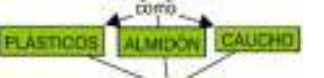
prosina apterarse en Reimanicos ion the

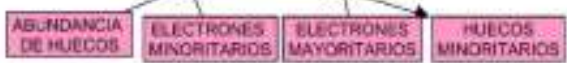
EMOPFOMEROS 


\section{Agradecimiento}

Agradecemos a la Dra. Lizeth Martínez Ayala, profesora de la Escuela Superior de Tepeji de la Universidad Autónoma del Estado de Hidalgo, por orientarnos en la realización del presente trabajo, siendo una guía fundamental para la publicación de esta trabajo.

\section{Referencias}

[1] Shanh, S., Kim, J., Tregger, N., \& Metaxa, Z. (2010). Proceedings of the Indian Concrete Institute Asian Conference on Ecstasy in Concrete. Proceedings of the Indian Concrete Institute Asian Conference on Ecstasy in Concrete, 1-8.

[2] Escudero, F. J. (1967). Terminología e interpretaciones físicas.

[3] I.E.S. Villalba Hervás. (2010). Tipos de materiales. Materiales de uso técnico, 1-11.

[4] Balvantin García, A. de J. (2010). Conceptos básicos, ciencia de materiales para ingeniería. 13. http://www.dicis.ugto.mx/profesores/balvantin/documentos/Cie ncia de Materiales para Ingenieria/Tema 1 - Conceptos Basicos.pdf

[5] Materiales, L. O. S., Propiedades, y., ciencia, L. A., \& Materiales, E. I. D. E. L. O. S. (n.d.). Los materiales, propiedades y aplicaciones.

[6] Dr.C. Reig. Tema 2 : Semiconductores intrínsecos y extrínsecos introducción. (n.d.). 1-13

[7] Aranzábal Olea, A. Semiconductores extrínsecos. (31 de 07 de 2020). Obtenido de semiconductores extrínsecos: http://www.sc.ehu.es/sbweb/electronica/elec_basica/tema2/Pag inas/Pagina6.htm

[8] Mecanismos de reciclado. (2017). Tema 2. Tipos de plásticos, reciclado, aditivación y mezclado. http://iq.ua.es/TPO/Tema2.pdf

[9] Piña, C. M. (2007). Los biomateriales y sus aplicaciones. Revista de osteoporosis y metabolismo mineral, 55-58. https://www.uv.es/materomo/resources/g11.pdf

[10] Duffó, G. S. (2012). Una mirada a los biomateriales. Instituto de Energía y Desarrollo Sustentable, 69-70.

[11] Calvo Santo-Rosa, A. (2013). Análisis y simulación numérica del proceso de biodegradación por hidrólisis de biopolímeros en aplicaciones biomédicas. Capítulo 2: Biomateriales. 5-16.

[12] Rogers, C. A. (1995). Materiales inteligentes. Investigación y ciencia (Scientific American), Noviembre (230), 1-5.

[13] Lopez, O., Carnicero, A., \& Ruíz, R. M. (2003). Materiales inteligentes $\{(\mathrm{I})\}$ Introducción a los materia les del siglo $\{\mathrm{XXI}\}$ Anales de mecánica y electricidad, LXXX (January), 40-46.

[14] Gaiker Centro Tecnológico. (22 de 08 de 2020). Gaiker, Member of basque research and technology alliance. Obtenido de http://www.gaiker.es/cas/materiales_inteligentes.aspx 